\title{
Observation of Atoms Laser Cooled below the Doppler Limit
}

\author{
Paul D. Lett, Richard N. Watts, Christoph I. Westbrook, and William D. Phillips \\ Electricity Division, National Bureau of Standards, Gaithersburg, Maryland 20899
}

Phillip L. Gould

Department of Physics, University of Connecticut, Storrs, Connecticut 06268

and

\author{
Harold J. Metcalf \\ Department of Physics, State University of New York at Stony Brook, Stony Brook, New York 11794
}

(Received 18 April 1988)

\begin{abstract}
We have measured the temperature of a gas of sodium atoms released from "optical molasses" to be as low as $43 \pm 20 \mu \mathrm{K}$. Surprisingly, this strongly violates the generally accepted theory of Doppler cooling which predicts a limit of $240 \mu \mathrm{K}$. To determine the temperature we used several complementary measurements of the ballistic motion of atoms released from the molasses.
\end{abstract}

PACS numbers: $32.80 . P \mathrm{j}, 42.50 . \mathrm{Vk}$

Laser cooling is the use of laser radiation to reduce the relative motion of a collection of atoms. The most common type of laser cooling is "Doppler cooling" where the Doppler shift causes the radiation pressure force to be velocity dependent, thus damping atomic motion when the laser frequency is tuned below an atomic resonance. Atoms (or ions) cooled by this method have been used in applications as diverse as high-resolution spectroscopy and studies of collisions at low energies. Other current and future applications of laser cooling include velocity compression and focusing of atomic beams, loading and cooling of atoms in traps, cooling and storage of antimatter, study of condensed phases such as Coulomb crystals and Bose condensates, and study of low-energy atom-surface interactions. For a review of laser cooling and its applications, see Phillips et al. ${ }^{1}$ In most such applications, the colder the atoms, the better.

Shortly after the idea for laser cooling of atoms ${ }^{2,3}$ appeared, it was realized ${ }^{4}$ that heating from random emissions and absorptions imposes a lower limit on the temperature. Several authors have worked out this theory. ${ }^{5-7}$ For low laser intensity, in one dimension (1D) or $3 \mathrm{D}$, the equilibrium temperature for free atoms is ${ }^{8}$

$$
k_{\mathrm{B}} T=\frac{\hbar \gamma}{4} \frac{1+(2 \Delta / \gamma)^{2}}{2|\Delta| / \gamma},
$$

where $\gamma$ is the natural width of the laser-driven transition and $\Delta<0$ is the detuning of the laser frequency from resonance. This temperature has a minimum value $k_{\mathrm{B}} T_{\mathrm{D}}=\hbar \gamma / 2$ when $\Delta=-\gamma / 2$. High power should lead to higher temperature. For the $D$ line of $\mathrm{Na}$, where $\gamma=2 \pi \times 10 \mathrm{MHz}, T_{\mathrm{D}}$, the "Doppler cooling limit," is 240 $\mu \mathrm{K}$.

For over ten years researchers in laser cooling have accepted the generality of $T_{\mathrm{D}}$ as a lower limit to Doppler cooling. A number of groups have indeed reported achievement temperatures at or near the cooling limit, both for free atoms ${ }^{9,10}$ and for trapped ions. ${ }^{11,12}$ Here we present experimental evidence for laser cooling of free atoms well below $T_{\mathrm{D}}$. These observations are both remarkable and unexpected, especially in light of the simplicity of the experiment. We have not used any of the number of schemes that have been proposed for the cooling of atoms below $T_{\mathrm{D}}{ }^{13-15}$ Our results are particularly significant because we have shown that it is relatively easy to achieve atomic temperatures much lower than previously thought possible. In fact, we believe that we have produced the coldest 3D gas ever observed.

We measure the temperature of a sample of sodium atoms cooled and confined in a region of optical molasses ${ }^{9,16,17}$ (so-called because the confinement is due to the strong viscous damping provided by laser cooling). Our molasses, loaded continuously with atoms from a laser-cooled atomic beam, ${ }^{16}$ is formed at the intersection of three orthogonal pairs of counterpropagating laser beams in a region where the field is $<20 \mu \mathrm{T}$. These are typically apertured to $9 \mathrm{~mm}$ diam and carry $5 \mathrm{~mW}$ in each of six beams. The frequency-stabilized laser providing these beams is tuned below resonance for the $\left(3 S_{1 / 2}, F=2\right) \rightarrow\left(3 P_{3 / 2}, F^{\prime}=3\right)$ transition. It contains $10 \%$ sidebands, $1732 \mathrm{MHz}$ from the carrier, the upper one of which reexcites any atoms pumped into the $\left(3 S_{1 / 2}, F=1\right)$ level. The linear polarization of each beam pair is orthogonal to that of the other pairs. This setup has been described elsewhere ${ }^{8}$ and is similar to that of Ref. 9.

We use four methods to determine the temperature, following this general sequence: With the molasses at steady state (density $\lesssim 10^{7} \mathrm{~cm}^{-3}$ ) its loading is shut off, and its density begins to decay (time constant $\$ 300 \mathrm{~ms}$, determined mainly by the rate of diffusion out of the molasses). After $20-50 \mathrm{~ms}$, before many atoms have escaped, all the molasses beams are turned off with a mechanical shutter (fall time to $10 \%$ is $30 \mu \mathrm{s}$, to com- 
plete extinction $40 \mu \mathrm{s}$ ) thereby releasing the atoms to travel ballistically in the dark.

The atomic temperature determines the distribution, in space and time, of the released atoms. Each of our four methods measures some feature of this distribution. The temperature is found by comparison of these measurements with calculations obtained by numerical integration over the assumed 3D Maxwell-Boltzmann velocity distribution of the atoms and the initial, measured spatial distribution of the atoms in the molasses. The calculations include such features as the considerable effect of gravity, the intensity distribution of the laser beams, and the geometry of the detection process.

The first method is a time-of-flight (TOF) determination of the temperature. A pair of counterpropagating beams, derived from the molasses laser (including the sidebands), form a probe region under the molasses. ${ }^{18}$ We vary the center-to-center separation $d$ from 1.0 to $1.9 \mathrm{~cm}$. The probe cross section is either a $4-\mathrm{mm}$-diam circle or a 4-mm-wide $\times 1-\mathrm{mm}$-high rectangle, with a power of $3 \mathrm{~mW}$ per beam. An $8.5-\mathrm{mm}$ length of probe, directly below the molasses, is imaged onto a photomultiplier, and the time dependence of the probe-induced fluorescence is recorded, after the molasses beams are shut off. Numerical calculations of this TOF signal show that the time of the peak signal is mainly sensitive to $d$ and to the temperature, but is relatively insensitive to other details.

Figure 1 shows a typical TOF signal, averaged for 40 repetitions, at $d=1.1 \mathrm{~cm}$, along with calculated signals. The data agree with the calculation for $40 \mu \mathrm{K}$ and are clearly inconsistent with the "cooling limit" of $240 \mu \mathrm{K}$. For these data $\Delta \cong-20 \mathrm{MHz}$; note that at this detuning Eq. (1) gives $510 \mu \mathrm{K}$. Figure 2 shows the variation in the measured temperature (determined from the time of the peak of the TOF signal) as a function of $\Delta$ for various $d$ 's. The data are radically different from Eq. (1): The temperature is minimized for $|\Delta|$ much larger than

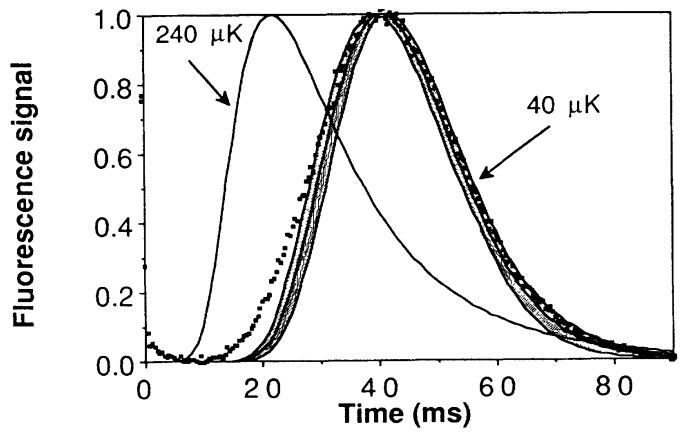

FIG. 1. TOF data and calculations. The shaded area indicates the range of error in the $40-\mu \mathrm{K}$ calculation from geometric uncertainties. The width of the data is slightly larger than the calculation, presumably because of shot-to-shot instabilities. the predicted $\gamma / 2$, and is always below the cooling limit except when $|\Delta| \lesssim \gamma / 2$. Also shown in Fig. 2 is the molasses decay rate. This was obtained from an exponential fit to the decay of the molasses fluorescence when the loading was shut off, but the molasses lasers were kept on. ${ }^{8}$ The following changes had no significant $(10 \mu \mathrm{K})$ effect on the measured temperature at $\Delta \cong-20 \mathrm{MHz}$ : reduction of the intensity of the molasses beams by a factor of 3 , or of the probe by a factor of 100 ; reduction of molasses density by a factor of 6; variation of the time between loading shutoff and molasses shutoff from 20 to $50 \mathrm{~ms}$. Considering the scatter between various measurements, uncertainties in the geometry, and the calculation, we determine the minimum temperature, with 1standard-deviation uncertainty, to be $43 \pm 20 \mu \mathrm{K}$.

In the second or "fountain" method the probe beam is placed a center-to-center height $h$ above the molasses. Atoms with a given upward velocity, released from the molasses, rise only to a given height in the gravitational potential. The number of atoms reaching the probe, as a function of $h$, is a measure of their temperature. Figure 3 shows the dependence of the time-integrated TOF signal on $h$, for $\Delta \cong-20 \mathrm{MHz}$. Error bars reflect statistical scatter and uncertainties due to baseline subtraction. $T=60 \mu \mathrm{K}$ best fits the data with $\chi^{2}=1.4$ for 4 degrees of freedom; $\chi^{2}$ for $240 \mu \mathrm{K}$ is 27.5 . The final result, with systematic and random errors is $60 \pm 25 \mu \mathrm{K}$.

In the third, or shower, method the probe is placed under the molasses and the time integral of the fluorescence is measured for various horizontal displacements of the probe. The horizontal distance traveled by the atoms while falling to the probe depends on their horizontal velocity. Thus, lower temperature will result in an integrated probe signal which decreases more rapidly as the probe is translated off center. For a vertical drop of



FIG. 2. $T$ vs $\Delta$ by TOF for various separations $d$. The solid curve represents the measured molasses decay rate; it is not a fit to the temperature data, but its scale was chosen to emphasize its proportionality to the temperature data. The dashed line shows the temperature expected on the basis of Eq. (1). 


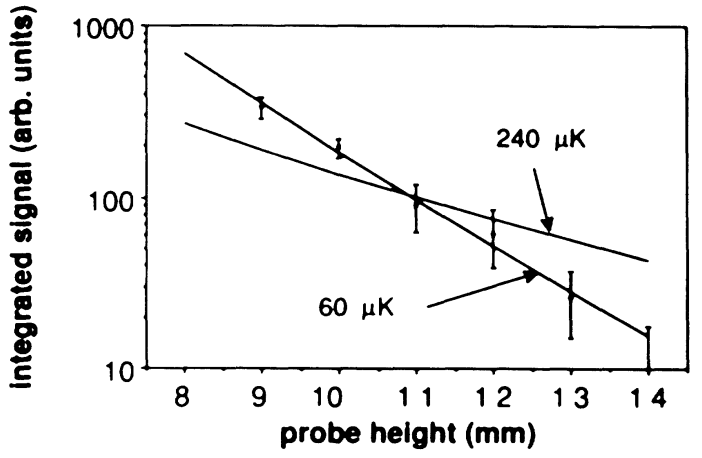

FIG. 3. Fountain method: measured signal vs probe height above molasses center, compared to calculations.

$1.1 \mathrm{~cm}$ and a detuning $\Delta \cong-20 \mathrm{MHz}$, our best-fit temperature is $80 \pm 25 \mu \mathrm{K}$ with a $\chi^{2}$ of 9.1 for 9 degrees of freedom. Our forcing the fit to $T=240 \mu \mathrm{K}$ gives $\chi^{2}$ $=89$, effectively ruling out a temperature as high as $T_{\mathrm{D}}$.

In the fourth method, release and recapture ( $R \& R$ ), no separate probe beam is used: The molasses beams are turned off for a time $t_{\text {off }}$, and then turned back on, recapturing the atoms still within the molasses region. From the molasses fluorescence we measure the fraction of atoms remaining as a function of $t_{\text {off }}$. This technique, used in the first experiments on optical molasses, ${ }^{9}$ gave $T=240 \pm 200 \mu \mathrm{K}$, consistent with the cooling limit in Na. Similar measurements consistent with the cooling limit have been made with cesium. ${ }^{10}$

To analyze our experiments, we have included the effects of gravity (quite important at $50 \mu \mathrm{K}$, but not at $240 \mu \mathrm{K}$ ), as well as nonuniform initial molasses distribution, and nonuniform intensity of the molasses lasers. For the case of a uniform, spherical molasses in the absence of a gravitational field, our numerical calculations of $\mathrm{R} \& \mathrm{R}$ agree with the analytic calculations in Ref. 9. Figure 4 shows (at $\Delta \cong-20 \mathrm{MHz}$ ) the measured fraction of atoms remaining as a function of $t_{\text {off. }}$. Calculated curves are also shown. The data give $T=45 \pm 20 \mu \mathrm{K}$. When an acousto-optic modulator (extinction ratio $=$ 500:1) was used to shut off the molasses in $<1 \mu \mathrm{s}$, the data were essentially unchanged. The $R \& R$ temperature as a function of $\Delta$ corroborates the dependence shown in Fig. 2.

We emphasize that the four methods described above are rather different in character and complementary in what they measure. This is important for elimination of questions about the low temperatures being the result of an experimental artifact. TOF measures the downward velocity component by measurements of the time for atoms to go between the molasses and the probe. $R \& R$ uses no probe and is sensitive to a composite of all velocity components. The fountain and shower methods depend entirely on gravity and do not use timing at all (the measured quantity is a time integral). The fountain measures upward velocity while the shower measures

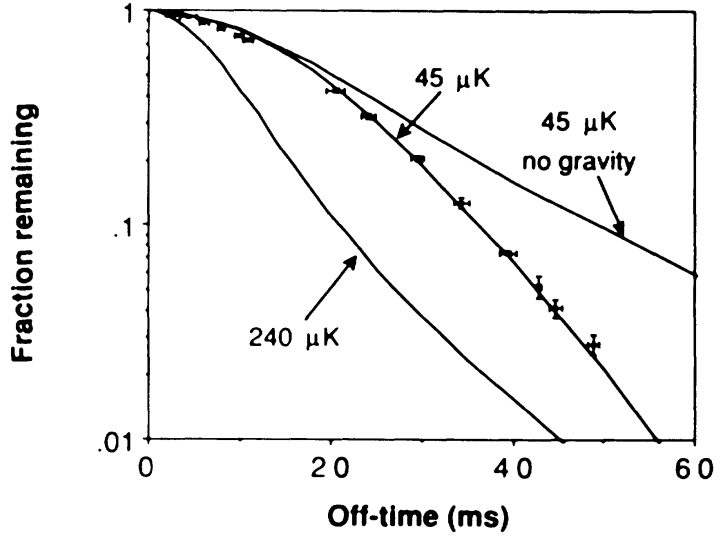

FIG. 4. Release and recapture: Measured ratio of total fluorescence from molasses before and after a period of darkness, $t_{\text {off }}$, compared with calculated ratios.

horizontal velocity. All methods have in common that they measure the temperature of atoms released from molasses, rather than of atoms confined in it. They all lead to the same conclusion: The temperature of the atoms is well below the Doppler cooling limit.

While we have no explanation for these surprising results, we offer several observations and speculations. The theory leading to Eq. (1) assumes a two-state atom and low laser power, and takes no account of laser polarization in 3D, or the presence of sidebands on the laser. A two-state, 1D theory, including higher power, ${ }^{6}$ yields a higher $T$. Using TOF at $\Delta \cong-20 \mathrm{MHz}$, we have observed that increasing the magnetic field from 0 to 0.2 $\mathrm{mT}$ increases $T$ by $120 \mu \mathrm{K}$. $T$ also depends on polarization: Rotation of the linear polarization of one pair of molasses beams by $70^{\circ}$ increases the temperature by 40 $\mu \mathrm{K}$. Thus, it may be that a multistate theory is needed to explain the results. Narrow Raman resonances or folded two-photon transitions among ground states might be involved in the cooling, although the independence of $T$ on beam power, which we saw in the TOF experiments, argues against many nonlinear processes. Alternately, a transient mechanism might provide cooling. However, any mechanism which altered the temperature upon release of the atoms would have to operate in less than $1 \mu \mathrm{s}$, our shortest turnoff time. Evaporative cooling (after the loading is turned off) does not explain the low temperature because so few atoms evaporate and the density is too low for collisional energy redistribution.

Earlier results, including the insensitivity of the molasses lifetime to intensity imbalance in a pair of beams,${ }^{8}$ the behavior of lifetime with detuning, ${ }^{8}$ and the strange properties of "super molasses" 19 were not consistent with existing theories. Figure 2 shows that $T$ and the molasses decay rate have the same dependence on $\Delta$. A diffusion analysis shows that the decay is proportional to $T / \alpha$, where $\alpha$ is the viscous damping constant. ${ }^{9}$ Thus, if molasses is described by simple diffusion, $\alpha$ is inde- 
pendent of $\Delta$, contradicting present theories. A large, constant $\alpha$ would account for the imbalance insensitivity and the low temperature (since $T \propto 1 / \alpha$ ). Also, atoms at these low temperatures, moving in a lattice of standingwave potential wells, ${ }^{19}$ might explain super molasses.

In conclusion, we have demonstrated a violation of the Doppler cooling limit in $\mathrm{Na}$ atoms released from optical molasses. Additional experiments must be performed, especially with different sideband conditions, and on atoms or transitions having different level structures from that used in these experiments (for example the $\mathrm{Na}$ $D 1$ line or the $F=1 \rightarrow F^{\prime}=0 D 2$ transition). Also, additional theoretical work is needed to extend the theory of laser cooling and optical molasses to cover the essential elements of 3D, multistate, multifrequency situations.

We thank the Office of Naval Research for its support. We also thank E. Williams, A. Migdall, D. Wineland, J. Hall, J. Dalibard, S. Chu, and C. Wieman for helpful discussions. Two of the authors (R.N.W. and C.I.W.) are National Bureau of Standards-National Research Council postdoctoral fellows.

Note added. - The group at the University of Colorado has recently measured the detuning dependence of the temperature and decay rate of optical molasses in cesium to be similar to what we have observed. ${ }^{20}$ Recent measurements at Stanford of the temperature of a $\mathrm{Na}$ molasses confirm that for sufficiently red detunings the temperature is well below the cooling limit. ${ }^{21}$

${ }^{1}$ W. Phillips et al., Science 239, 877 (1988), and references therein.

${ }^{2}$ T. Hänsch and A. Schawlow, Opt. Commun. 13, 68 (1975).

${ }^{3}$ D. Wineland and H. Dehmelt, Bull. Am. Phys. Soc. 20, 637
(1975).

${ }^{4}$ V. Letokhov et al., Zh. Eksp. Teor. Fiz. 72, 1328 (1977) [Sov. Phys. JETP 45, 698 (1977)]; E. Purcell, private communication, cited in Ref. 5; W. Neuhauser et al., Phys. Rev. Lett. 41, 233 (1978).

${ }^{5}$ D. Wineland and W. Itano, Phys. Rev. A 20, 1521 (1979).

${ }^{6} \mathrm{~J}$. Gordon and A. Ashkin, Phys. Rev. A 21, 1606 (1980).

${ }^{7}$ R. Cook, Phys. Rev. A 22, 1078 (1980).

${ }^{8} \mathrm{P}$. Gould et al., in Laser Spectroscopy VIII, edited by S. Svanberg and W. Persson (Springer-Verlag, Berlin, 1987), p. 64. [Note that the denominator should be squared in Eq. (3).]

${ }^{9}$ S. Chu et al., Phys. Rev. Lett. 55, 48 (1985).

${ }^{10} \mathrm{D}$. Sesko et al., to be published.

${ }^{11}$ W. Nagourney et al., Proc. Natl, Acad. Sci. USA 80, 643 (1983).

${ }^{12}$ J. Bergquist et al., Phys. Rev. A 36, 428 (1987).

${ }^{13}$ D. Pritchard, Phys. Rev. Lett. 51, 1336 (1983).

${ }^{14}$ S. Chu et al., Opt. Lett. 11, 73 (1986).

${ }^{15}$ H. Metcalf, Bull. Am. Phys. Soc. 33, 913 (1988).

${ }^{16}$ W. Phillips et al., J. Opt. Soc. Am. B 2, 1751 (1985).

${ }^{17} \mathrm{~J}$. Bjorkhholm et al., in Advances in Laser Science-II -1986, edited by W. C. Stwalley et al., AIP Conference Proceedings No. 160 (American Institute of Physics, New York, 1987), p. 319.

${ }^{18} \mathrm{We}$ use counterpropagating probes to avoid accelerating the atoms out of resonance before they scatter many photons. Even so, the heating from spontaneous emissions perpendicular to the probe quickly "boils" the atoms out, limiting the time they spend in the probe. We have used this effect to create a quasidirectional atomic beam by cutting off one or two of the molasses beam pairs, "squirting" the molasses atoms out perpendicular to the remaining beam(s) with typical velocities of $120 \mathrm{~cm} / \mathrm{s}$. This technique should be useful in the making of atomic fountains and other slow beams.

${ }^{19}$ S. Chu et al., in Ref. 8, p. 58.

${ }^{20} \mathrm{C}$. Wieman, private communication.

${ }^{21} \mathrm{~S}$. Chu, private communication. 


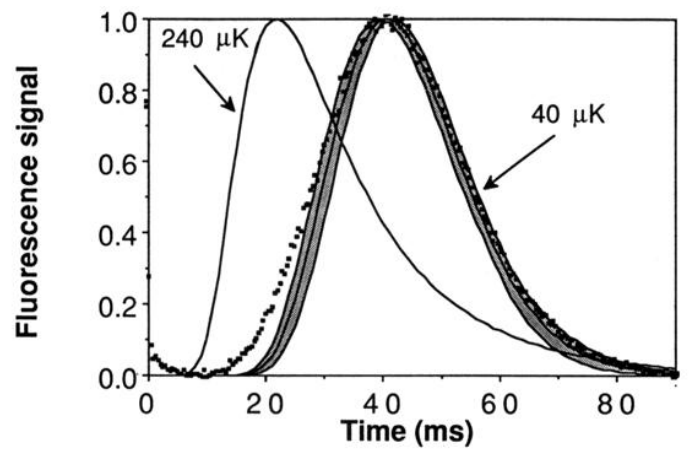

FIG. 1. TOF data and calculations. The shaded area indicates the range of error in the $40-\mu \mathrm{K}$ calculation from geometric uncertainties. The width of the data is slightly larger than the calculation, presumably because of shot-to-shot instabilities. 